\title{
Time and Cost of Hospitalisation for Salvage Therapy in Adults with Philadelphia Chromosome-Negative B Cell Precursor Relapsed or Refractory Acute Lymphoblastic Leukaemia in Spain
}

\author{
Blanca Boluda ${ }^{1} \cdot$ Rebeca Rodríguez-Veiga $^{1}$ - David Martínez-Cuadrón ${ }^{1,2} \cdot$ Ignacio Lorenzo $^{1}$ - Jaime Sanz ${ }^{1,2}$. \\ Ana Regadera ${ }^{1} \cdot$ Amparo Sempere $^{1,2} \cdot$ Leonor Senent ${ }^{1} \cdot$ Jose Vicente Cervera $^{1,2} \cdot$ Pilar Solves $^{1,2} \cdot$ John Reitan $^{3}$. \\ Salvador Gea ${ }^{4}$ Miguel Angel Sanz ${ }^{1,2}$ Pau Montesinos ${ }^{1,2}$
}

Published online: 15 October 2018

(c) The Author(s) 2018

\begin{abstract}
Background Philadelphia chromosome-negative $(\mathrm{Ph}-)$ relapsed or refractory $(\mathrm{R} / \mathrm{R}) \mathrm{B}-$ cell precursor acute lymphoblastic leukaemia (ALL) is rare, and information on its impact on healthcare systems is scarce.

Objective To quantify the time and reimbursement associated with hospitalisations of patients with R/R ALL in a Spanish hospital.

Methods Retrospective review of medical charts identified patients aged $\geq 18$ years with $\mathrm{Ph}-\mathrm{R} / \mathrm{R}$ ALL hospitalised between 1998 and 2014. Data were collected from the date of first diagnosis of R/R ALL (index) until death or loss to follow-up. The primary endpoint was the proportion of time hospitalised during chemotherapy. Reimbursement associated with hospitalisations (including associated chemotherapy) was also assessed.

Results Thirty-two patients were eligible for inclusion. Their median age was 41 years, and $50 \%$ had a first remission duration of $\leq 1$ year; $34 \%$ had undergone allogeneic haematological stem-cell transplantation (alloHSCT). Overall, 31 patients had received intensive salvage chemotherapy, during which there were 42 hospitalisations (mean 1.4/patient; mean duration 26 days). Patients spent a mean of $71 \%$ of the chemotherapy period in hospital. Total mean reimbursement was $€ 26,417$ per patient, almost all $(€ 25,723)$ attributable to inpatient stays $(€ 18,986 /$ hospitalisation). From the index date to death or loss to follow-up (excluding alloHSCT-related hospitalisations), there were 80 hospitalisations (mean duration 24 days); mean reimbursement was $€ 16,692$ per hospitalisation and $€ 41,730$ per patient. AlloHSCT $(n=8)$ involved 18 hospitalisations (mean reimbursement $€ 39,782 /$ hospitalisation; $€ 89,510 /$ patient).

Conclusion Data from this sample of patients suggest that hospitalisations in R/R ALL are lengthy and associated with high costs in Spain.
\end{abstract}

\section{Key Points for Decision Makers}

The data suggest that hospitalisations for relapsed/refractory acute lymphoblastic leukaemia are common in Spain and often last for several weeks.

Such hospital admissions are associated with high costs.

\section{Introduction}

La Fe, Avinguda de Fern ando Abril Martorell, 106, 46026 Valencia, Spain

2 CIBERONC, Instituto Carlos III, Madrid, Spain

3 RJM Group, Crown Point, IN, USA

4 Amgen Health Economics, Barcelona, Spain
Philadelphia chromosome-negative $(\mathrm{Ph}-) \mathrm{B}$-cell precursor relapsed or refractory $(\mathrm{R} / \mathrm{R})$ acute lymphoblastic leukaemia (ALL) in adults is a rare condition; its projected incidence 
in Spain is 80-85 new cases per year [1]. Adult patients with $\mathrm{Ph}-\mathrm{B}$-cell precursor R/R ALL represent a relatively young population, having a median age of 34-39 years [2]. For patients who do not respond (are refractory) to therapy with curative intent or whose disease returns (relapses) following successful response to treatment (i.e. those with $\mathrm{R} / \mathrm{R}$ disease), salvage chemotherapy is the standard of care. This refers to chemotherapy offered in the absence of any other effective treatment options. Historically, there has been no universally accepted salvage chemotherapy regimen for adults with $\mathrm{Ph}-\mathrm{R} / \mathrm{R}$ ALL $[3,4]$, and these patients have a poor prognosis. Three-year survival rates range from 4 to $11 \%$ depending on important prognostic factors, including the number of previous therapies and duration of the first period of disease remission in response to treatment [2].

In Spain, the most common salvage chemotherapy for R/R ALL is FLAG-IDA (fludarabine, cytarabine, granulocyte colony-stimulating factor and idarubicin), an acute myeloid leukaemia-type chemotherapy scheme that results in high myelotoxicity, leading to an elevated risk of treatment-related infection and mortality [5-7]. Side effects of treatment and the poor condition of patients combine to leave individuals in need of extensive hospitalisation during therapy. Management of these patients requires considerable effort; however, the associated hospital and financial burdens have not been extensively documented in Spain. Understanding the reimbursement associated with standard therapies can help physicians and payers to put the costs of new therapies into context. The objective of this study was to quantify the time and reimbursement associated with hospitalisations for R/R ALL treatment in a Spanish hospital.

\section{Methods}

\subsection{Patients}

Patients eligible for inclusion in the study were aged at least 18 years and had been hospitalised for the management of at least one episode of R/R ALL. Patient charts for individuals with $\mathrm{Ph}-\mathrm{B}$-cell precursor ALL who were consecutively diagnosed with R/R ALL between 1998 and 2014 in Hospital Universitari i Politècnic La Fe (Valencia, Spain) were identified. Data for patients who met one of the following criteria were used in the analysis: had relapsed, with the first remission lasting less than 12 months; had relapsed after the first salvage therapy; had relapsed at any time after haematological stem-cell transplantation (HSCT) from a matched donor (allogeneic; alloHSCT); or were refractory to initial (primary induction) or salvage therapy. Patients were excluded from the study if they were enrolled in blinatumomab- or inotuzumab ozogamicin-related clinical trials during the study period.

\subsection{Study Design}

This was a single-centre retrospective chart review with a study period from 1998 to 2014. Patients were screened from 2014, going backwards to include all eligible individuals. The protocol was approved by the Research Ethics Board of the hospital, in accordance with the principles of the Declaration of Helsinki.

Individual patient data were collected from the date of first diagnosis of R/R ALL (index date) until death or loss to follow-up. The index date was defined as the first date on which the individual was recorded as having R/R ALL in their medical records. The cut-off date for the analysis was January 2016; the target number of patients to be included was at least 30 .

The chemotherapy period was defined as the time from the date of the first chemotherapy session after the index date to whichever occurred first out of death, loss to followup, last chemotherapy dose plus 30 days or initiation of alloHSCT. Only patients for whom a salvage chemotherapy regimen was recorded were included in the analysis of the chemotherapy period (Fig. 1).

For patients who underwent alloHSCT after the index date, the alloHSCT period was defined as the time from starting alloHSCT to whichever occurred first out of death, loss to follow-up or relapse (Fig. 1).

\subsection{Outcomes}

The primary outcome investigated was the percentage of time spent hospitalised as an inpatient during the chemotherapy period. This was calculated by dividing the number of inpatient days by the total number of days of the chemotherapy period. Secondary outcomes included frequency, types and durations of hospitalisations; reasons for hospitalisations; and reimbursement for hospitalisations. These outcomes were related to the number of hospital admissions and were calculated for the chemotherapy period. The same

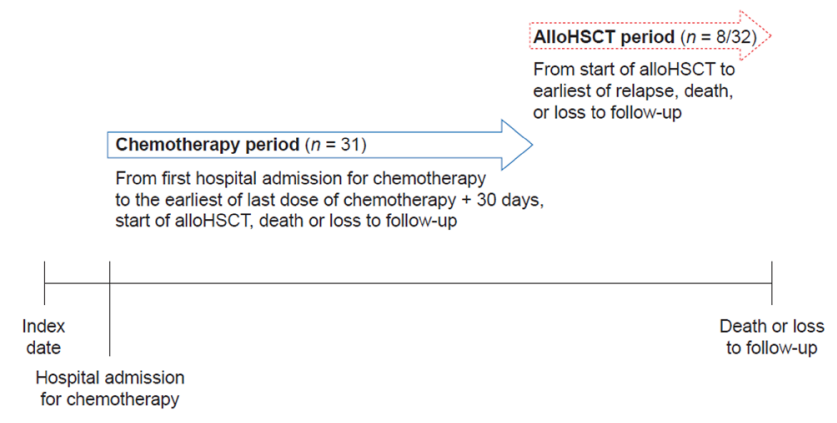

Fig. 1 Study schema. AlloHSCT allogeneic haematopoietic stem-cell transplantation 
analyses were calculated from the index date until death or loss to follow-up, including and excluding the alloHSCT period.

\subsection{Calculation of Hospital Reimbursement}

The national diagnosis-related group (DRG) list provides information on the amount of money reimbursed to the hospital and the average length of stay for each DRG code. Reimbursement for chemotherapy does not need to be added to the costs because it is considered to be included in the reimbursement for the assigned DRG code. Reimbursement followed the algorithm for haematology hospitalisations at the centre. The reimbursement for outpatient care was $€ 108$ for each outpatient visit and $€ 269$ for each day's stay at a day hospital; these values were based on the mean day hospital unit costs calculated using costs from all Spanish regions [8]. Briefly, DRG codes (2016) [9] and corresponding reimbursements were assigned as follows, with only one DRG code assigned for each hospitalisation (Fig. 2): DRG $576(€ 27,274)$ if the overnight stay included or was due to induction with intensive chemotherapy; DRG $577(€ 12,444)$ if the overnight stay included or was due to consolidation or intensification using intensive chemotherapy; DRG 577 $(€ 12,444)$ if the overnight stay was not related to administration of intensive chemotherapy but was associated with a major complication (e.g. sepsis, pneumonia, parenteral nutrition or respiratory failure); DRG 876 (€4475) if the overnight stay was not related to administration of intensive chemotherapy or associated with a major complication (e.g. administration of chemotherapy and discharge when the schedule was completed, or hospitalisation due to any non-major complication). For alloHSCT, the DRG code 803 was assigned and was associated with a reimbursement of $€ 60,599$ plus $€ 692$ for each day beyond 40 days [8].

For some hospitalisations, the length of stay was longer than the mean given in the DRG list; those cases were assumed to be associated with major complications, and the DRG code 577 was used.

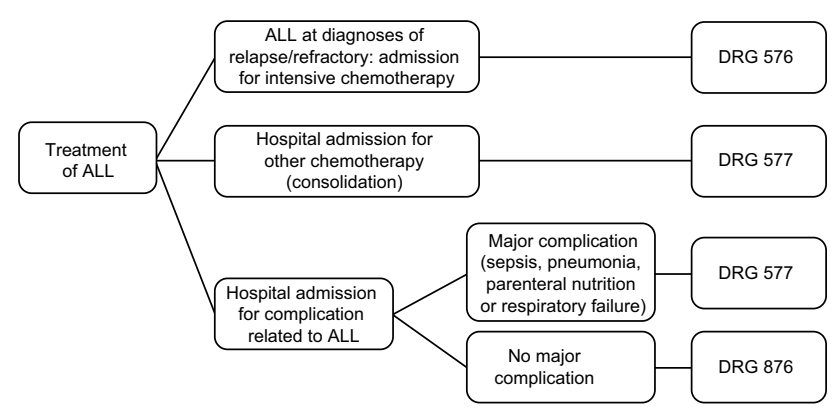

Fig. 2 Decision algorithm for assignment of DRG code for hospitalisation episodes. $A L L$ acute lymphoblastic leukaemia, $D R G$ diagnosisrelated group

\subsection{Statistical Analyses}

The target sample size of at least 30 patients was set as a practical and achievable study population, on the basis of experience with this rare disease in the Hospital Universitari i Politècnic La Fe. Data for all patients who met the inclusion criteria were included in the analyses. Formal statistical comparisons between groups were not carried out; descriptive statistics were calculated for all outcomes. Means (with standard deviation [SD]) or medians (interquartile range and range) were provided for continuous variables, and incidences and proportions were provided for categorical variables.

\section{Results}

\subsection{Patients}

Thirty-two patients with $\mathrm{Ph}-\mathrm{B}$-cell precursor R/R ALL were eligible for inclusion in the study, following exclusion of 21 ineligible cases (four $<18$ years old; five with $\mathrm{Ph}+$ status; 12 with a remission lasting at least 1 year). The median age was 41 years, and $34 \%$ of patients were men. Half of the patients $(n=16)$ had a first remission lasting 12 months or less, 11 (34\%) relapsed after alloHSCT, four $(13 \%)$ were refractory to salvage chemotherapy, and one (3\%) relapsed after first salvage. Most patients (88\%) had died by the end of the study period (Table 1).

\subsection{Hospitalisations and Reimbursement During the Chemotherapy Period}

Thirty-one patients received intensive salvage chemotherapy after the index date and were included in the analysis of the chemotherapy period: FLAG-IDA in 13 patients (42\%), FLAG-IDA plus another regimen in ten patients $(32 \%)$ and other intensive induction schedules in eight patients $(26 \%)$. The median duration of the chemotherapy period was 68 days (range 34-72 days). During this time, there were 42 inpatient hospitalisations with a mean duration of 26 days, and the mean number of hospitalisations per patient was 1.4 (Table 2). Regarding the primary outcome, patients spent a mean of $71 \%$ (95\% confidence interval [CI] 61-82) of the chemotherapy period in hospital. Mean reimbursement was $€ 26,417$ per patient; the vast majority of this total $(€ 25,723)$ was attributable to inpatient stays ( $€ 18,986 /$ hospitalisation). Day visits and outpatient visits accounted for 23 and 52 hospitalisations, respectively (Table 2 ). 
Table 1 Patient characteristics

\begin{tabular}{lc}
\hline Characteristic & $N=32$ \\
\hline Age at index date, years & $41(30-58)$ \\
Median (range) & $43(17)$ \\
Mean (SD) & $21(66)$ \\
Women, $n(\%)$ & \\
Disease status at index date, $n(\%)$ & $16(50)$ \\
Relapsed with first remission $\leq 12$ months & $1(3)$ \\
Relapsed after first salvage (with first remission > 12 months) & $11(34)$ \\
Relapsed any time after alloHSCT & $4(13)$ \\
Refractory to primary induction or salvage therapy & \\
Status at the end of follow-up, $n(\%)$ & $28(88)$ \\
Deceased & $4(13)$ \\
Alive (lost to follow-up) & \\
Treatment received, $n(\%)$ & $31(97)$ \\
Chemotherapy & $8(25)$ \\
AlloHSCT & \\
\hline
\end{tabular}

AlloHSCT allogeneic haematopoietic stem-cell transplantation, $S D$ standard deviation

Table 2 Hospitalisations and reimbursement during the chemotherapy period

\begin{tabular}{llll}
\hline & Inpatient hospitalisations & Day visits & Outpatient visits \\
\hline $\begin{array}{l}\text { Per hospitalisation } \\
\text { Number of hospitalisations }\end{array}$ & 42 & 23 & 52 \\
Mean (SD) length of stay, days & $26(18.0)$ & $1(0.0)$ & $1(0.0)$ \\
Mean (SD) reimbursement, $€$ & $18,986(9272)$ & $691(0)$ & $108(0)$ \\
Per patient & & & \\
Number of patients & 31 & 31 & 31 \\
Mean (SD) number of stays & $1.4(1.0)$ & $0.7(1.9)$ & $1.7(3.0)$ \\
Mean (SD) length of stay, days & $36(29.0)$ & NA & NA \\
Mean (SD) reimbursement, $€$ & $25,723(16,957)$ & $513(1324)$ & $181(329)$ \\
\hline
\end{tabular}

$N A$ not applicable, $S D$ standard deviation

\subsection{Hospitalisations and Reimbursement from the Index Date to Death or Loss to Follow-Up (Excluding AlloHSCT)}

From the index date to death or loss to follow-up, there were 80 inpatient hospitalisations (excluding those for alloHSCT), with a mean duration of 24 days. Patients spent a mean of $57 \%$ (95\% CI 48-66) of this period (excluding alloHSCT) in hospital. The mean reimbursement was $€ 43,753$ per patient, and again almost all reimbursements $(€ 41,730)$ were attributable to inpatient stays ( $€ 16,692 /$ hospitalisation). During this period, there were 63 days visits and 196 outpatient visits (Table 3 ).

\subsection{Hospitalisations and Reimbursement for Patients who Underwent AlloHSCT}

Eight patients underwent alloHSCT (Table 4). The mean length of hospital stay for alloHSCT was longer (33 days), and reimbursement per inpatient hospitalisation was higher $(€ 39,782)$ than for the chemotherapy treatment period. Patients spent a mean of 39\% (95\% CI 25-53) of the chemotherapy period in hospital. AlloHSCT hospitalisation was associated with a mean reimbursement of $€ 89,510$ per patient (Table 4).

\subsection{Reasons for Hospitalisation}

Reasons for inpatient hospitalisation at any time during the study period were assessed; more than one reason for each hospitalisation was possible. The most common primary reasons for hospitalisation were receipt of chemotherapy (56\%), fever (28\%), sepsis (27\%) and neutropenia (24\%) (Fig. 3); blood transfusion was a contributing cause of hospitalisation for most patients (77\%). Reasons with a frequency of less than $10 \%$ could not be meaningfully grouped according 
Table 3 Hospitalisations and reimbursement during the period from the index date to death or loss to follow-up (excluding alloHSCT)

\begin{tabular}{llll}
\hline & Inpatient hospitalisations & Day visits & Outpatient visits \\
\hline Per hospitalisation & & & \\
Number of hospitalisations & 80 & 63 & 196 \\
Mean (SD) length of stay, days & $24(19.0)$ & $691(0)$ & $1(0.0)$ \\
Mean (SD) reimbursement, $€$ & $16,692(9473)$ & & \\
Per patient & & 32 & 32 \\
Number of patients & 32 & $2.0(3.1)$ & $6.1(7.5)$ \\
Mean (SD) number of stays & $2.5(1.6)$ & NA & NA \\
Mean (SD) length of stay, days & $59(37.0)$ & $1361(2133)$ & $662(815)$ \\
Mean (SD) reimbursement, $€$ & $41,730(21,282)$ & & \\
\hline
\end{tabular}

AlloHSCT allogeneic haematological stem-cell transplantation, $N A$ not applicable, $S D$ standard deviation
Table 4 Hospitalisations and reimbursement during the alloHSCT period

\begin{tabular}{llll}
\hline & Inpatient hospitalisations & Day visits & Outpatient visits \\
\hline Per hospitalisation & & & \\
$\quad$ Number of hospitalisations & 18 & 60 & 61 \\
Mean (SD) length of stay, days & $33(30)$ & $1(0)$ & $1(0)$ \\
Mean (SD) reimbursement, $€$ & $39,782(34,228)$ & $691(0)$ & $108(0)$ \\
Per patient & & & \\
Number of patients & 8 & 8 & 8 \\
$\quad$ Mean (SD) number of stays & $2.3(1.4)$ & $7.5(11.1)$ & $7.6(7.2)$ \\
$\quad$ Mean (SD) length of stay, days & $73(34)$ & NA & NA \\
Mean (SD) reimbursement, $€$ & $89,510(21,379)$ & $5186(7646)$ & $824(781)$ \\
\hline
\end{tabular}

AlloHSCT allogeneic haematological stem-cell transplantation, $N A$ not applicable, $S D$ standard deviation

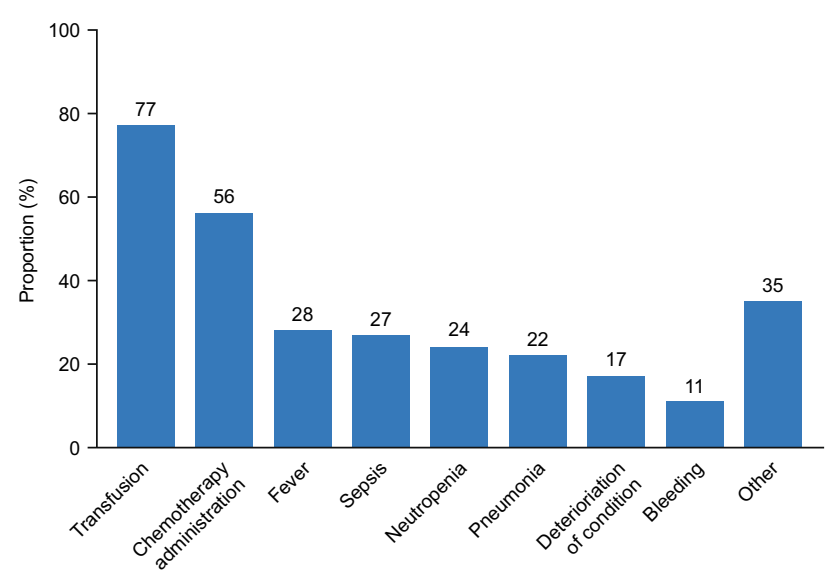

Fig. 3 Reasons for inpatient hospitalisation during the study period. Reasons with a frequency of $10 \%$ or more are shown; all others are grouped together in the 'Other' category. Patients could be hospitalised for more than one reason. Note that transfusion was not a primary reason for hospitalisation

to clinical criteria, owing to a broad range of reasons being documented, and were therefore not included separately in the analysis. Instead, these reasons were included in the 'Other' category (Fig. 3).

\section{Discussion}

This single Spanish institution study indicates that the standard treatment for the analysed period in adult patients with $\mathrm{R} / \mathrm{R}$ ALL is associated with lengthy hospitalisations and high costs.

After R/R ALL was diagnosed, the majority of adult patients received salvage chemotherapy. These patients spent almost three-quarters of their treatment time in hospital. Hospitalisations were associated with chemotherapy administration and/or major complications, resulting in high DRG-associated costs.

In other retrospective chart reviews conducted in Italy [10], France [11] and Germany [12] (with study populations of 26-39 patients), the investigators reported slightly lower proportions of patient time (46-63\%) spent hospitalised during the chemotherapy period. Although not all of the supporting information is available in these reports, one can speculate that the other countries (especially, France [11]) may experience a greater degree of outpatient management than Spain. Differences between countries in the 
drugs selected for salvage regimens might also be expected to impact on the extent of hospitalisation. FLAG-IDA was the preferred salvage regimen in our single-centre study; this regimen is associated with prolonged neutropenia and frequent extra-haematological toxicities, requiring inpatient management [13]. In general, patients were admitted to hospital for more than one reason, most commonly chemotherapy and infection-related complications, as was found in other studies [10-12].

Regarding the cost burden on the healthcare system, our data show high costs, in line with those observed in other patient chart reviews conducted in Europe [10-12] and in the USA [14]. The costs reported in this Spanish hospital are lower than those seen in other retrospective chart studies. Reimbursement during the chemotherapy period was $€ 18,986$ per hospitalisation in our study compared with $€ 23,568$ in Italy, €30,301 in Germany and €31,067 in France [10-12]. Similarly, reimbursement during the alloHSCT period reported here was $€ 39,782$, compared with $€ 74,366$ in Italy, $€ 67,696$ in Germany and $€ 43,672$ in France [10-12].

The prognosis of patients with R/R ALL is generally poor, with a reported median overall survival of less than 6-7 months [2, 4, 6]. Under these circumstances, time spent in hospital and quality of life (QoL) should be considered as relevant treatment outcomes. Recently, the impact of salvage therapy on QoL was analysed in the phase 3, randomised, open-label TOWER study comparing blinatumomab with a pre-selected standard chemotherapy regimen [15]. In the standard-of-care arm, rapid deteriorations in QoL were observed as early as day 8 of the first treatment cycle, while statistically significant improvements in almost all functional scale scores analysed were observed in the experimental arm.

New immunotherapies have improved the response rates that are achievable in adult patients with R/R ALL [16, 17], providing a bridge to potentially curative alloHSCT. It remains unclear, however, whether these agents change the natural history of the disease.

To our knowledge, this is the first report of hospitalisations and reimbursement associated with the treatment of adult patients with $\mathrm{Ph}-\mathrm{R} / \mathrm{R}$ ALL in Spain. However, as well as being subject to the general bias and restrictions of a retrospective analysis, the study has a number of other limitations. Although the analysis involves a relatively large series of adults with R/R ALL and the sample size is similar to that in other European retrospective chart reviews, the number of patients included is too small to draw generally applicable conclusions about the burden on healthcare resources of treating R/R ALL. Furthermore, the results are taken from a single institution, in contrast to multicentre European studies, raising the question of whether our results can be extrapolated to other centres across Spain. However, the standard of care in our institution followed current Spanish guidelines for the treatment of adult patients with R/R ALL (i.e. FLAG-IDA followed by alloHSCT). Given that our study covers a period of 16 years, there may have been heterogeneity in patient management and costs over this time. Unfortunately, the standard of care remained unchanged at our institution from 1998 to 2014. Finally, the costs used to populate this analysis were based on data from a Spanish online resource of economic data for the healthcare sector to support the DRG codes [8]. Benchmarking the cost data used in this study against an alternative source of Spanish hospital costs data revealed that costs were broadly similar for DRG 576 ( $€ 27,274$ vs $€ 26,837$ ), DRG 577 ( $€ 12,444$ vs $€ 9156)$ and DRG 876 ( $€ 4475$ vs $€ 4740$ ), but varied substantially for DRG 803 ( $€ 60,599$ vs $€ 35,436$ ). Although this comparison indicates that there are some differences in costs between different sources, in general there is an encouraging level of consistency suggesting that our costing model was valid [18].

\section{Conclusion}

In summary, this study suggests that standard salvage therapy for adult patients with $\mathrm{Ph}-\mathrm{B}$-cell precursor R/R ALL is associated with lengthy hospitalisations and high costs in Spain.

Acknowledgements Medical writing support was provided by James O'Kelly, an employee of Amgen, and Kim Allcott, from Oxford PharmaGenesis, Oxford, UK. Guillermo Sanz, from the Hospital Universitari i Politècnic La Fe, Valencia and Instituto Carlos III, Madrid, provided support with the study design, data analysis and drafting the manuscript. Vincenzo Vella, an employee of Amgen, provided support in data analysis and drafting the latest versions of the manuscript.

Data Availability Statement We agree on providing the dataset, software code and/or model underpinning our research.

Author contributions JR and SG designed the study. BB, RR-V, DM-C, IL, JS, AR, AS, LS, JC, PS, JR, MS and PM contributed to the data acquisition. BB, RR-V, DM-C, IL, JS, AR, AS, LS, JVC, PS, JR, SG, MAS and PM performed the data analysis. All authors were involved in drafting and revising the manuscript and provided their approval for its publication. PM will act as the overall guarantor and corresponding author.

\section{Compliance with Ethical Standards}

Funding This study and medical writing support for this article were sponsored by Amgen (Europe) $\mathrm{GmbH}$. This work was partially financed with Fonds Européen de Développement Économique et Régional (FEDER) funds (CIBERONC CB16/12/00284).

Conflict of interest BB has no disclosures. RRV has no disclosures. DMC has no disclosures. IL has no disclosures. JS has no disclosures. AR has no disclosures. AS has no disclosures. LS has no disclosures. JVC has no disclosures. PS has no disclosures. JR is an employee of 
RJM Group; RJM received funding from Amgen to conduct this study. SG is an employee of Amgen and holds stock in Amgen Inc. MAS has no disclosures. PM has received remuneration from Amgen for a speaker bureau.

Ethical approval The protocol was approved by the Research Ethics Board of the hospital, in accordance with the principles of the Declaration of Helsinki.

Informed consent For this type of study, formal consent is not required.

Open Access This article is distributed under the terms of the Creative Commons Attribution-NonCommercial 4.0 International License (http://creativecommons.org/licenses/by-nc/4.0/), which permits any noncommercial use, distribution, and reproduction in any medium, provided you give appropriate credit to the original author(s) and the source, provide a link to the Creative Commons license, and indicate if changes were made.

\section{References}

1. Katz AJ, Chia VM, Schoonen WM, Kelsh MA. Acute lymphoblastic leukemia: an assessment of international incidence, survival, and disease burden. Cancer Causes Control CCC. 2015;26(11):1627-42.

2. Gokbuget N, Dombret H, Ribera JM, Fielding AK, Advani A, Bassan R, et al. International reference analysis of outcomes in adults with B-precursor Ph-negative relapsed/refractory acute lymphoblastic leukemia. Haematologica. 2016;101(12):1524-33.

3. Saltman D, Barlev A, Seshagiri D, Katsoulis I, Lin V, Barber B. Management and treatment of relapsed or refractory $\mathrm{Ph}(-)$ B-precursor ALL: a web-based, double-blind survey of EU clinicians. BMC Cancer. 2015;15:771.

4. Hoelzer D, Bassan R, Dombret H, Fielding A, Ribera JM, Buske C. Acute lymphoblastic leukaemia in adult patients: ESMO clinical practice guidelines for diagnosis, treatment and follow-up. Ann Oncol. 2016;27(Suppl 5):v69-82.

5. Bergua JM, Montesinos P, Martinez-Cuadron D, Fernandez-Abellan P, Serrano J, Sayas MJ, et al. A prognostic model for survival after salvage treatment with FLAG-Ida \pm gemtuzumab-ozogamicine in adult patients with refractory/relapsed acute myeloid leukaemia. Br J Haematol. 2016;174(5):700-10.

6. Dominguez Senin L, Rodriguez Rodriguez JN, Garrido Martinez MT, Sanchez Argaiz M, Martin Chacon E. Effectiveness and safety of the FLAG-IDA regimen in acute refractory or recurrent leukaemia. Farmacia hospitalaria: organo oficial de expresion cientifica de la Sociedad Espanola de Farmacia Hospitalaria. 2012;36(4):261-7.
7. Specchia G, Pastore D, Carluccio P, Liso A, Mestice A, Rizzi $\mathrm{R}$, et al. FLAG-IDA in the treatment of refractory/relapsed adult acute lymphoblastic leukemia. Ann Hematol. 2005;84(12):792-5.

8. Oblikue Consulting. eSalud-Información económica del sector sanitario. http://esalud.oblikue.com/. Accessed 14 Mar 2018.

9. Ministry of Health and Social Services and Equity. Instituto de Información Sanitaria (2018). Registro de altas-CMBD 2016. http://pestadistico.inteligenciadegestion.msssi.es/publicoSNS/ comun/DefaultPublico.aspx. Accessed 14 Mar 2018.

10. Pagano L, Oberti M, Esposito B, Reitan J, Barlev A, Barber $\mathrm{BL}$, et al. Retrospective chart review of hospitalisations during chemotherapy for adult patients with $\mathrm{Ph}$-negative B-precursor relapsed or refractory $(\mathrm{R} / \mathrm{R})$ acute lymphoblastic leukaemia (ALL) in Italy. Value Health J Int Soc Pharmacoecon Outcomes Res. 2015;18(7):A443

11. Dombret H, Thomas X, Chevallier P, Nivot E, Reitan J, Barber B, et al. Healthcare burden and reimbursement of hospitalization during chemotherapy for adults with Ph-negative relapsed or refractory B-cell precursor acute lymphoblastic leukemia in France: a retrospective chart review. J Med Econ. 2016;19(11):1034-9.

12. Kreuzer K, Stuhlmann R, Lebioda A, Reitan J, Barber BL, Barlev A. Hospitalisations among adult patients with $\mathrm{Ph}$-negative B-precursor relapsed or refractory (R/R) acute lymphoblastic leukaemia (ALL) receiving chemotherapy in Germany: a retrospective chart review. Value Health J Int Soc Pharmacoecon Outcomes Res. 2015;18(7):A443.

13. de la Rubia J, Regadera A, Martin G, Cervera J, Sanz G, Martinez $\mathrm{J}$, et al. FLAG-IDA regimen (fludarabine, cytarabine, idarubicin and G-CSF) in the treatment of patients with high-risk myeloid malignancies. Leuk Res. 2002;26(8):725-30.

14. Barlev A, Lin VW, Song X. Burden of hospitalization in relapsed acute lymphoblastic leukemia. Curr Med Res Opin. 2016;32(7):1209-12.

15. Topp MS, Zimmerman Z, Cannell P, Dombret H, Maertens J, Schuh AC, et al. Health-related quality of life (HRQoL) of blinatumomab versus standard of care (SOC) chemotherapy in patients with relapsed or refractory Philadelphia negative B-cell precursor acute lymphoblastic leukemia in a randomized, open-label phase 3 study (TOWER). Blood. 2016;128(22):222.

16. Kantarjian H, Stein A, Gokbuget N, Fielding AK, Schuh AC, Ribera JM, et al. Blinatumomab versus chemotherapy for advanced acute lymphoblastic leukemia. N Engl J Med. 2017;376(9):836-47.

17. Kantarjian HM, DeAngelo DJ, Stelljes M, Martinelli G, Liedtke $\mathrm{M}$, Stock W, et al. Inotuzumab ozogamicin versus standard therapy for acute lymphoblastic leukemia. N Engl J Med. 2016;375(8):740-53.

18. Red Española de Costes Hospitalarios (RECH). https://recho sp.org. Accessed 27 Feb 2018. 